\title{
DEVELOPMENT OF A MORE REALISTIC SAILING SIMULATOR
}

\author{
Jonathan R. Binns ${ }^{1}$, j.binns@mte.amc.edu.au, \\ Frank W. Bethwaite ${ }^{2}$, frank@ bethwaite.com, \\ Norman R. Saunders ${ }^{3}$, n.saunders@ unimelb.edu.au.
}

\begin{abstract}
New software has been developed for an existing simulator. The physical model of the simulator has been rewritten and now includes a lot of extra features which have increased the realism of the simulation considerably. The new physical model has been explained in this paper and compared with the old model.
\end{abstract}

$\begin{array}{ll}\text { NOMENCLATURE } \\ A & \text { area } \\ A R & \text { geometric aspect ratio } \\ c & \text { chord } \\ C_{D} & \text { drag coefficient } \\ C_{L} & \text { lift coefficient } \\ \rho & \text { fluid density } \\ t & \text { time } \\ V & \text { velocity }\end{array}$

\section{INTRODUCTION}

The forerunner of the Virtual Sailing VS-1 simulator was originally developed for sports physiology, the science of studying athletic performance. Accurate measurement of an athlete's performance requires that he or she exercises in the laboratory. To study sailors it was necessary to invent a "marine treadmill". Over more than a decade this early design was developed into a comprehensive sailing simulator by the Department of Anatomy and Physiology in the School of Medicine of the University of Tasmania (Walls et al [13]).

Virtual Sailing then redesigned and rebuilt the laboratory machine. Compared to the original University of Tasmania simulator (see Gale and Walls [4]), the current VS-1 hardware utilises a complete Laser (or other dinghy) hull suspended on rollers. This not only gives the user a more realistic feel, because they are sitting in an actual dinghy, but also gives a much more realistic feel to the roll, because the centre of roll is much closer to where it would be in real life. Another major difference between the VS-1 and the original simulator is in the pneumatics. The VS-1 has very high specification pneumatics, resulting in a very quick and powerful pneumatic response.

The VS-1 enables a non-threatening introduction to sailing as well as a training platform for more advanced sailors. Tuition takes place day and night in all weathers. Tacking, gybing, sail and boat trim can all be taught in a realistic, dynamic environment. The VS-1 consists of a Laser class or similar hull mounted in an actuator frame. Pneumatic rams enable the hull to roll in response to the simulated conditions. The simulator is controlled by means of a rudder and a spring/sensor device provides sail trim information and "mainsheet feel".

The VS-1 simulator has been touring the world for the last two years and considerable feedback has been obtained from current and potential users. Numerous upgrade options have been proposed, but before any of these could be implemented it became apparent that a complete rebuild of the software side of the simulator was required.

The software rebuild has taken two programmers six months to complete. A total of over 40,000 new lines of code needed to be written, leaving virtually no stone unturned. All aspects of the visual representations, physical modelling and data acquisition systems have been rewritten to allow for future upgrades. Complete modularisation of the code has turned pipedream upgrades into reality.

In the process of rewriting the physical modelling it became apparent that the original program had numerous fundamental problems. With the application of some rudimentary velocity prediction techniques these fundamental problems have been put under control, the way forward is now much clearer.

This paper will describe this relatively simple model and compare it with the original physical model. It is shown that with the new model, a far more realistic simulation is obtained.

\section{THE SIMULATOR}

The simulator consists of a computer, a screen, a dinghy hull, a data acquisition system, some position indicators and two pneumatic rams. The whole system has been on show at Earl's Court where it was sailed by all grades of sailor from novice to elite as illustrated in Figure 1.

\footnotetext{
${ }^{1}$ Research Assistant, Faculty of Maritime Transport \& Engineering, Australian Maritime College.

${ }^{2}$ Chairman/Director, Bethwaite Design Pty Ltd. (also Director of Virtual Sailing Pty Ltd)

${ }^{3}$ Professorial Fellow, Department of Pharmacology, University of Melbourne (also Director of Virtual Sailing Pty Ltd)
} 


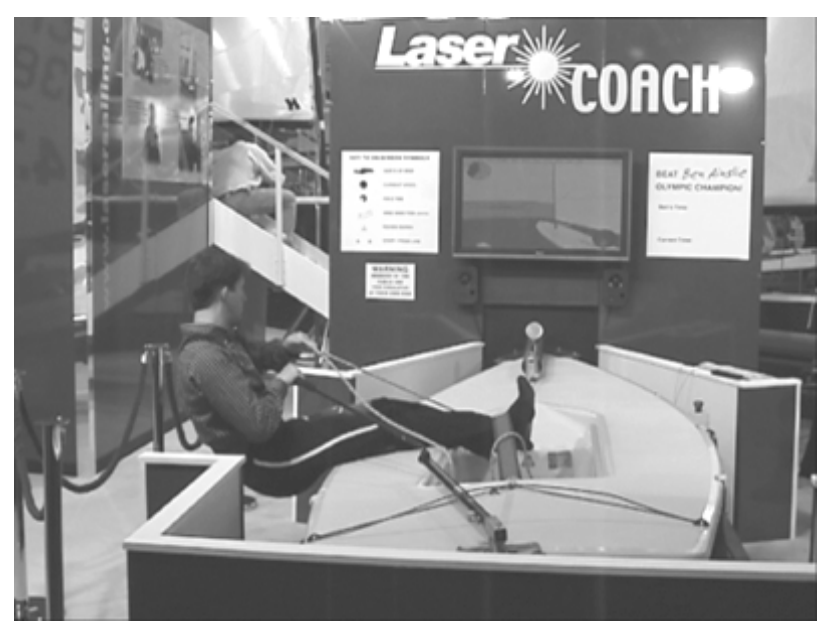

Figure 1. The Sailing Simulator at Earl's Court

Essentially the simulator provides a unique "human-inthe-loop" simulation, with control shared between the user, the simulator and the central computer. A schematic diagram of the feedback loop is shown in Figure 2.

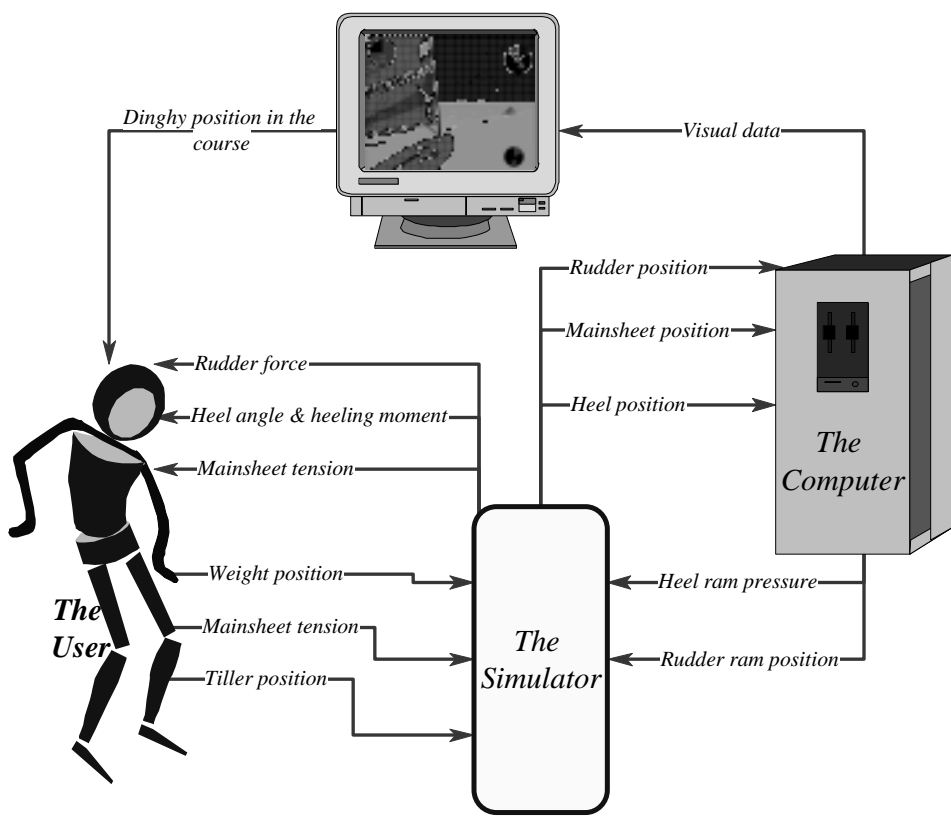

Figure 2. The simulator feedback loop

In Figure 2 the user receives feedback from the visual representation of where the dinghy is relative to the wind and course, as well as feedback from the simulator in the form of rudder forces, mainsheet tension, heel moment and angle. It is then up to the user to balance these forces and moments. As the forces and moments are applied via pneumatic rams and a spring, the feel of this real force balance is quite realistic. The simulator then takes the results of these real force balances through linear and potentiometer transducers and feeds them into the computer. The computer then carries out the bulk of the simulation, based on a relatively simple velocity prediction model. This numerical approach then yields a new dinghy position, which is fed back to the visual representation, and a new heel moment and rudder force, fed back to the simulator.

\section{THE PHYSICAL MODEL}

Three basic boats have been programmed. Data for these boats have been obtained from dinghy class data presented on the internet.

- The Laser see http://www.laserinternational.org. In addition three sail options have been coded, these are the standard sail, the radial sail and the 4.7 sail. For these different sail options the total area, centre of pressure, chord and aspect ratio have all been coded to make effects based on these plan form area characteristics.

- The Byte see http://www.blackdogmarine.com .

- The Optimist see http://www.optiworld.org .

The characteristics of these three dinghies have been coded into the new program, described in this paper. The visual representations of these boats are shown in Figure 3, Figure 4 and Figure 5.

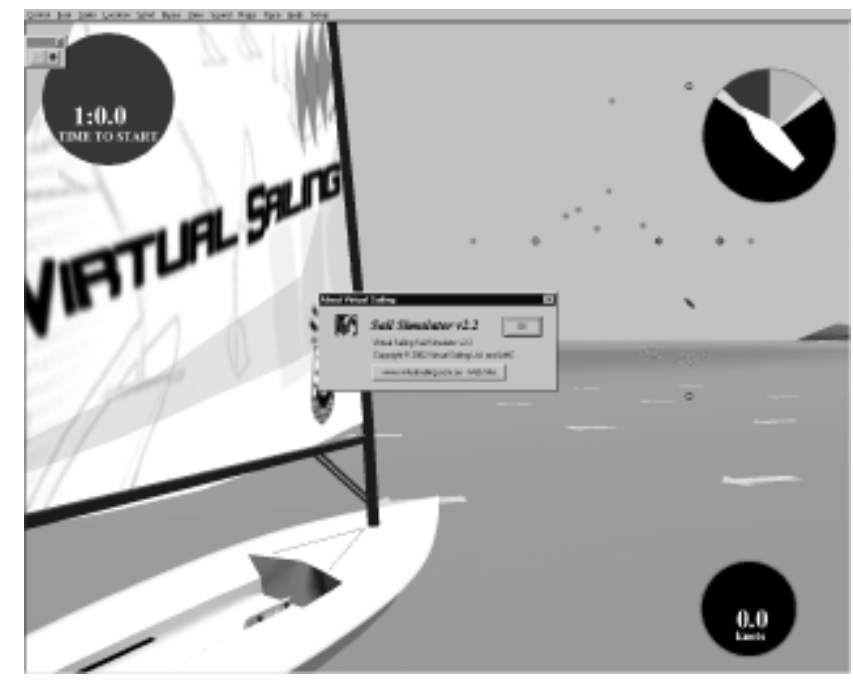

Figure 3: Byte visual representation

This figure illustrates the computer screen view as seen by the helm (or spectators). The dinghy image is a representation of a Byte. The boom and sail move when the actual sheet is pulled in or allowed out by the helm. Sail trim is indicated by sail flogging and a tell-tale. Arrows on the water indicate wind direction. Lower right icon is a boat velocity indicator; upper right gives position of hull relative to the wind; upper left is elapsed time from start of race. In the "sky' is an overhead view of a race course (buoys, starting marks, rocks and boat position). 


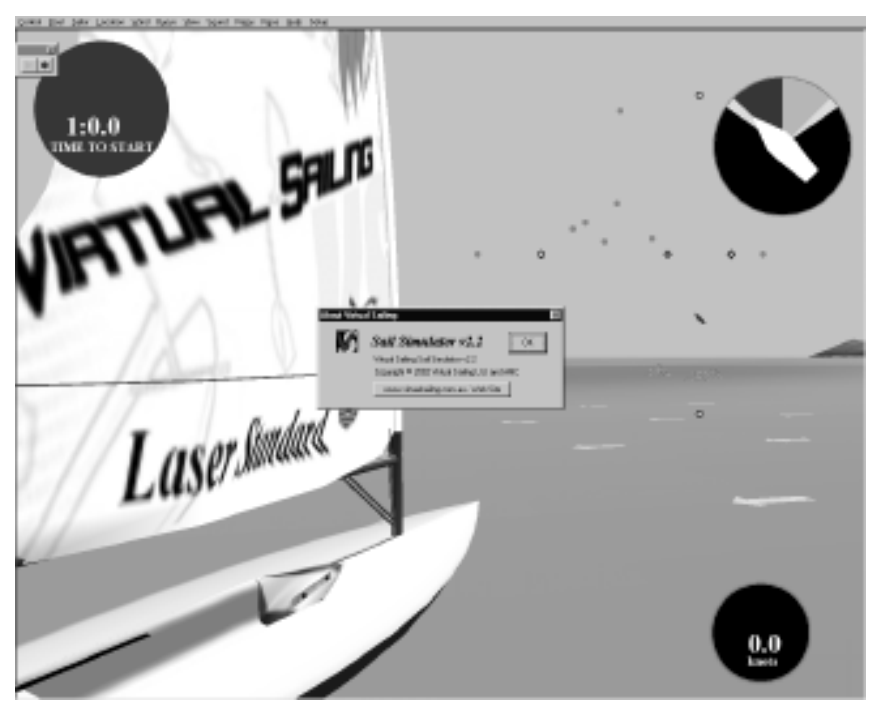

Figure 4: Laser visual representation

Detail as in Figure 3.

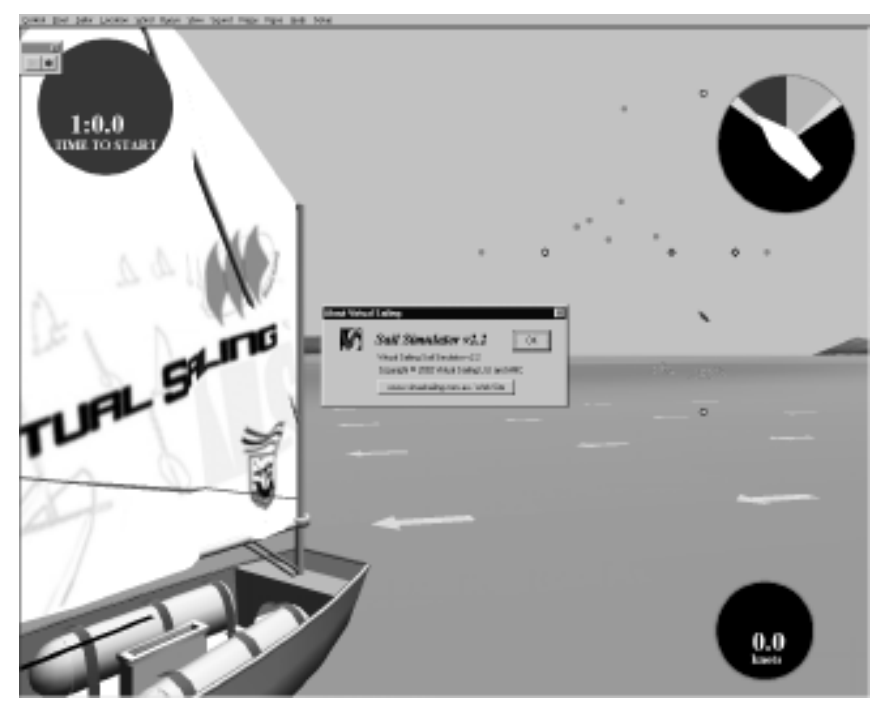

Figure 5: Optimist visual representation

Detail as in Figure 3.

\subsection{Force and moment balance}

In earlier versions of the simulator the force and moment balance was restricted to a forward force balance, a heel moment balance and a yaw moment balance. The lack of a side force balance meant that some force and moment items appear to have been assigned values based on subjective assessments of the simulator performance. For example the rudder force was obtained from a linear heel moment into yaw moment term, which meant, amongst other things, that at zero heel angle rudder forces would be zero regardless of mainsheet position.

The new program has a side force balance explicitly added to the simulation. This has made simulation of many more sailing phenomena possible, for example very low speed performance of the simulator is much more realistic. The new program also added a rudimentary vertical force balance, which only really affects the visual representation occasionally, and the ability to add the pitch degree of freedom has been coded, in advance of further physical model developments.

\subsection{Upright drag}

In the absence of experimental data, the best method of determining upright drag is to use a regression formula such as that shown in Gerritsma et al [5].

The final as-used upright drag curves are shown in Figure 6.

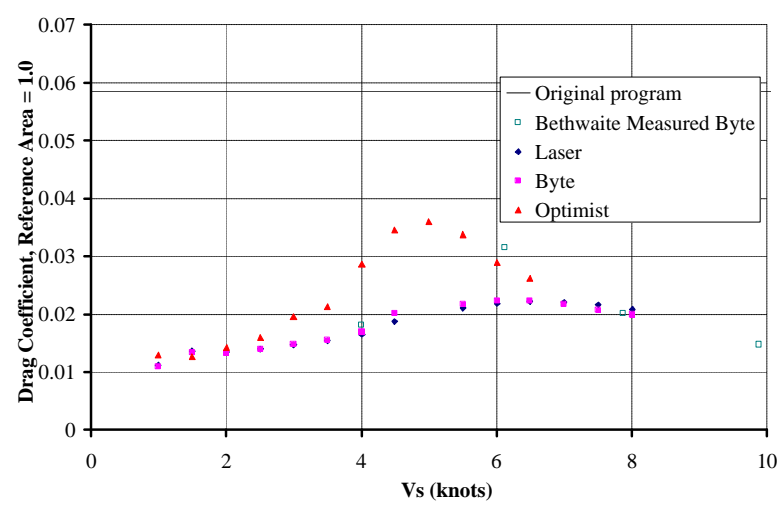

Figure 6: Final as-used upright drag curves

In Figure 6, the vertical axis is the upright drag coefficient (or area $\left(\mathrm{m}^{2}\right)$ ), obtained by dividing the upright drag by $0.5 \rho V^{2}$, where $\rho$ is the water density $\left(\mathrm{kg} / \mathrm{m}^{3}\right)$ and $V$ is the flow velocity $(\mathrm{m} / \mathrm{s})$. The horizontal axis is the flow velocity in knots. Although this representation of the data is not dimensionally sound, it has become useful in yacht research in the past to reduce errors of interpretation, see Teeters [12].

Also plotted in Figure 6 are the values for the original constant drag coefficient and that measured by Frank Bethwaite.

\subsection{Sail lift and drag}

The sail lift and drag polars were obtained from Marchaj p 587 [7]. These were then modified to take the varying geometric aspect ratios of the sails into account. The resultant curves of lift and drag are shown below. 


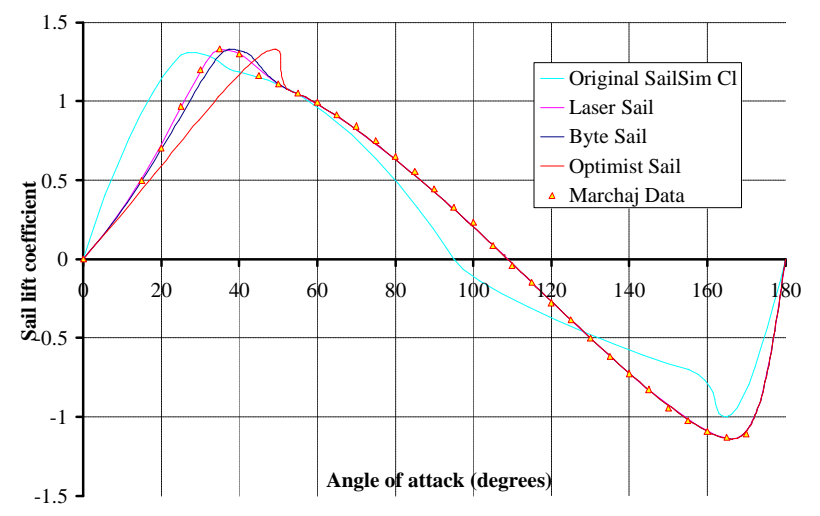

Figure 7: Sail lift coefficients

In Figure 7, the vertical axis is the sail lift coefficient, obtained by taking the component of the total force on the sail perpendicular to the wind velocity and dividing it by $0.5 \rho V^{2} A$, where $\rho$ is the air density $\left(\mathrm{kg} / \mathrm{m}^{3}\right), V$ is the wind velocity $(\mathrm{m} / \mathrm{s})$ and $A$ is the sail planform area $\left(\mathrm{m}^{2}\right)$. The horizontal axis is the angle of attack of the sail.

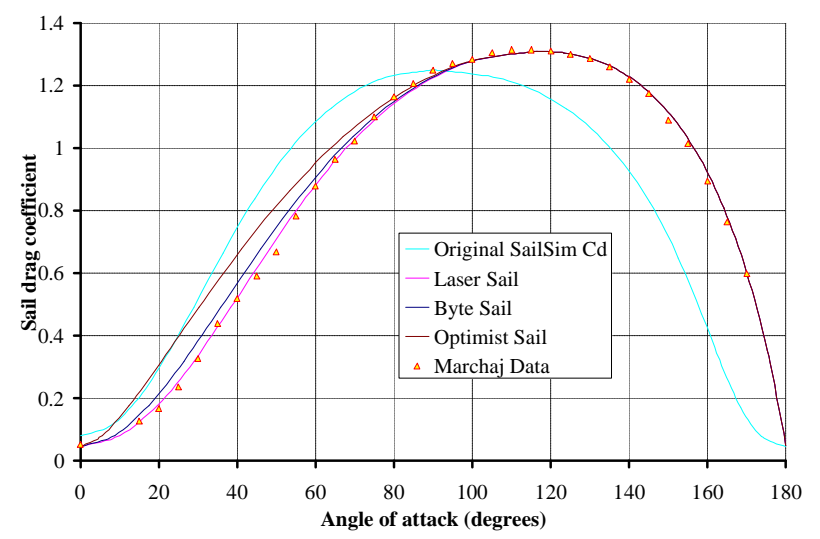

Figure 8: Sail drag coefficients

In Figure 8, the vertical axis is the sail drag coefficient. The horizontal axis is the angle of attack of the sail.

\subsection{Daggerboard lift and drag}

The daggerboard (either rudder or centreboard) lift and drag values were taken from Lewis pp 306-307 [6]. These were also adjusted for aspect ratio in the same way as for the sail. The results for the foils have been shown below.

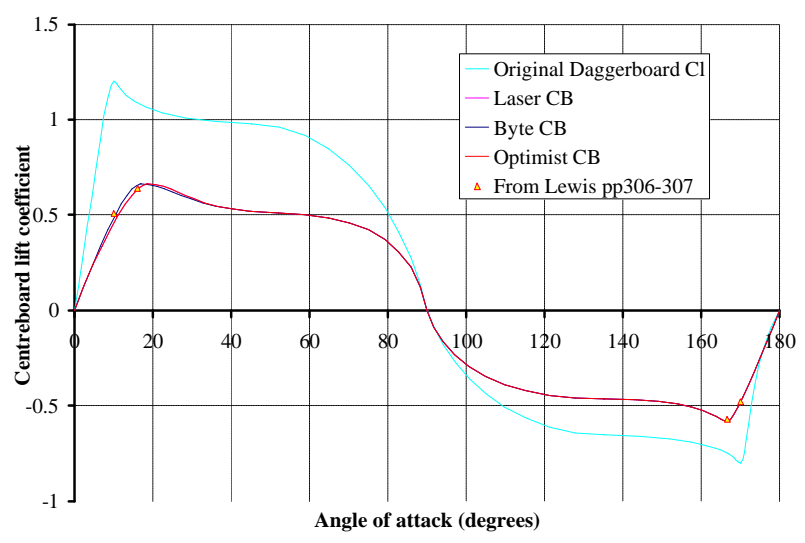

Figure 9: Centreboard lift coefficients

In Figure 9, the vertical axis is the centreboard lift coefficient, where the area, $A$, is the centreboard planform area $\left(\mathrm{m}^{2}\right)$. The horizontal axis is the angle of attack of the centreboard.

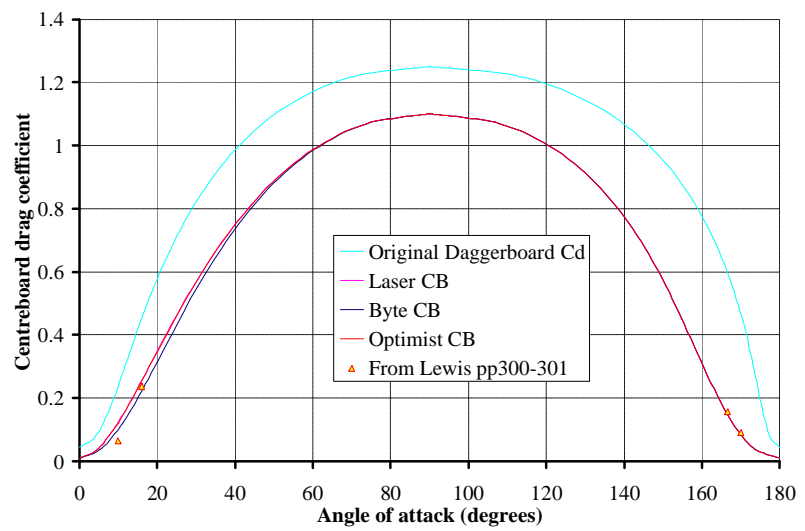

Figure 10: Centreboard drag coefficients

In Figure 10, the vertical axis is the centreboard drag coefficient. The horizontal axis is the angle of attack of the centreboard.

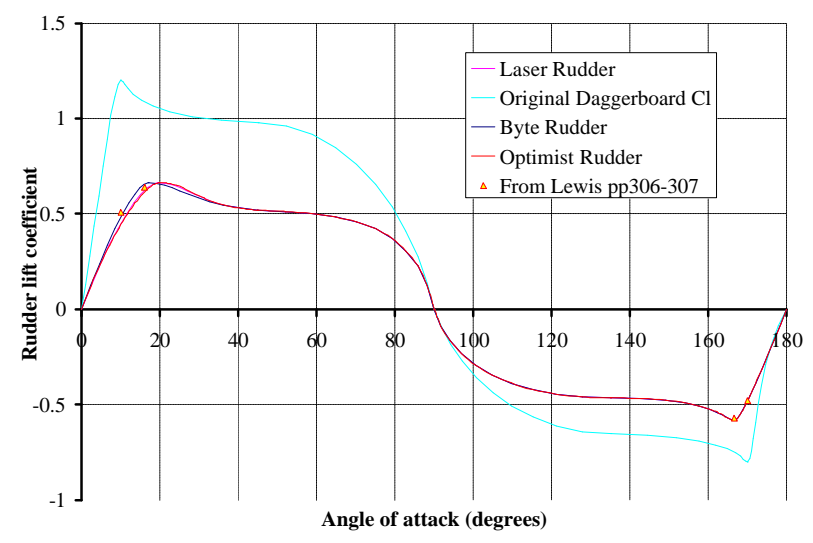

Figure 11: Rudder lift coefficients

Detail as in Figure 9. 


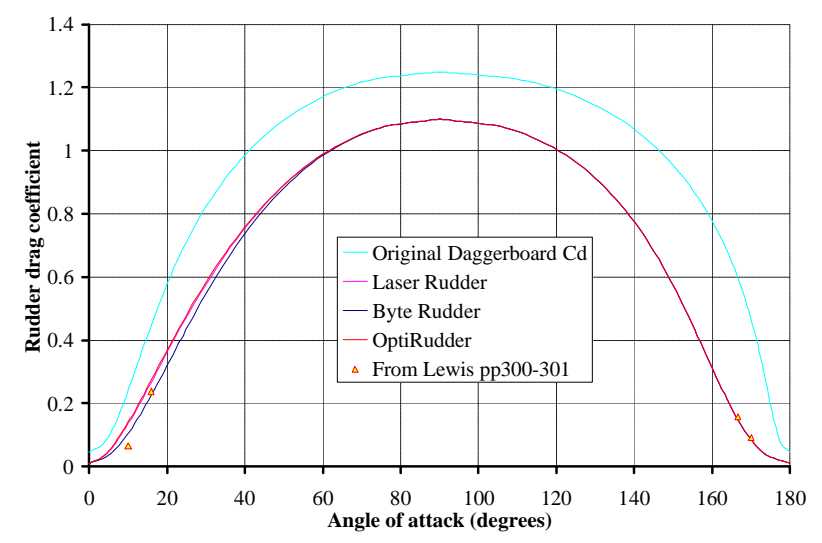

Figure 12: Rudder drag coefficients

Detail as in Figure 10.

\subsection{Effect of aspect ratio}

The lift and drag curves presented above have been incorporated into the simulation by fitting the data with bezier curves. The effect of aspect ratio has been introduced by shifting the control points of these bezier curves. The amount that the control points have been shifted is in proportion to the ratio of the actual geometric aspect ratio to the base geometric aspect ratio. The base geometric aspect ratio for the sail data was 3.7, for the daggerboards it was 2.13.

The drag and lift ratios used were based on inviscid theory, see Rae and Pope pp 240-241 [8], and come out to be

$$
\begin{aligned}
\text { Drag ratio } & =\frac{C_{D, A C T U A L}}{C_{D, B A S E}}, \\
& =\frac{A R_{B A S E}}{A R_{A C T U A L}} \\
\text { Lift ratio } & =\frac{C_{L, A C T U A L}}{C_{L, B A S E}} \\
& =\frac{1+\frac{2}{A R_{B A S E}}}{1+\frac{2}{A R_{A C T U A L}}}
\end{aligned}
$$

where $C_{L}$ is the lift coefficient, $C_{D}$ is the drag coefficient, $\mathrm{AR}_{\mathrm{BASE}}$ is the base geometric aspect ratio and $\mathrm{AR}_{\mathrm{ACTUAL}}$ is the actual geometric aspect ratio.

\subsection{Time dependent lift build up}

When a lifting surface attains an angle of attack there is a substantial time delay until the full lift is produced. This phenomenon can have a large effect on controllability when large attack angles are set suddenly. Therefore a simple model has been coded which follows the model proposed in Marchaj p 206 [7]. This model proposes that the time taken to reach $90 \%$ of the full lift can be calculated by

$$
t=\frac{6 c}{V}
$$

where $t$ is the time taken, $c$ is the chord length of the lifting surface and $V$ is the flow velocity. Put another way the foil will have to travel 6 chord lengths at a particular angle of attack before $90 \%$ of the maximum lift is reached.

It has then been assumed that sufficient realism can be obtained by using a linear increase in lift in time (as opposed to the hyperbolic curve shown in Marchaj).

During software trials it was expressed that there appears a slightly greater time lag in the rudder response resulting in occasional oversteering. This is probably due to the fact that the current program has a much greater update rate for the calculations and the visuals as compared with the old program, and yet the data acquisition systems are still tied to the slower update rate. This means that the rudder angle will change slower than what the operator might expect from the visual display. In an attempt to cure some of this problem the time dependent lift build up for the rudder has been reduced. The best fix for this problem would be to investigate upgrading the hardware.

\subsection{Static cross-coupling}

Through the use of three-dimensional geometry what might be termed static cross-coupling has been achieved. The most obvious phenomenon this affects is in the heel into yaw cross coupling, which is apparent to the user as windward helm in the rudder. This is currently achieved solely by considering the geometry of the sail, rudder and keel. This very simple approach works very well.

\subsection{Dynamic coefficients}

All dynamic coefficients (the added mass and damping terms) have been calculated and implemented based on published experimental data. The published data have been used by non-dimensionalising with respect to critical boat parameters, so changes in boat parameters will change the dynamic coefficients in a rudimentary manor. These could be refined in the future, but it would require considerable research to achieve this, and it would result in only a relatively small improvement in simulator performance.

Data have been obtained from Saunders pp 419-420 [11], Lewis pp 61-63 [6] and Bertram p 157 [1].

\subsection{Dynamic cross-coupling}

During an evaluation of the new software it was decided that a much higher degree of realism was required in the yaw moment balance. After experimentation with some critical parameters it appeared that such an increase in realism would require substantial dynamic crosscoupling. A reduced version of the suggested crosscoupling was implemented and can be summarised as follows:

\footnotetext{
- a linear sway velocity into yaw moment term;
} 
- a linear heel angle and quadratic surge velocity into yaw moment term; and

- the yaw velocity feeds into the angle of attack on the rudder.

The first two effects have been calculated using experimental data presented in Renilson et al [10], Binns [3] and Renilson and Manwarring p 455 [9]. The last term has been calculated from pure geometry assuming that the boat will be yawing about the centreboard.

It is the authors' opinion that using these scientifically based coefficients has improved the simulator considerably.

\subsection{Tiller sculling}

During an evaluation of the software the ability of the boat to get stuck in irons now was highlighted as a potential operational problem. This problem has arisen because the new simulation not only allows the boat to stop but will actually allow it to sail in reverse (which requires reversed steering).

A solution was suggested whereby tiller sculling could be modelled. This has been implemented by retaining the previous time step rudder angle. Then by differentiating this with the current rudder angle, a rudder angular velocity can be obtained. Then this has been incorporated by assuming that the effect can be simplified by converting that angular velocity into an average increase in cross flow over the rudder, simply added to the current flow velocity vector over the rudder. These assumptions appear to work well enough and it is now relatively easy to get the boat out of irons by sculling with the rudder.

\subsection{Gust simulation}

A gust model was derived based on the data presented in Bethwaite pp 36-37 [2]. In Bethwaite it is stated that there is about a 60 second period between gusts, which corresponds to a spatial separation of $200 \mathrm{~m}$ in 6 knots and $700-1000 \mathrm{~m}$ in 20 knots. This means that the gusts have to be travelling at around 6.5 knots and 22.732.4 knots in the 6 knot and 20 knot winds respectively. From this and from feedback on software evaluations it was decided that gust velocity should be set at $80 \%$ of the average wind speed.

Also in Bethwaite it is stated that gust diameters are usually not less than $100-200 \mathrm{~m}$. Due to the small nature of the course used it was decided to scale this value down, such that when the "Big Course" option is used the gust diameter is $90 \mathrm{~m}$ and the gust spacing is $300 \mathrm{~m}$. If the standard, small, course option is used then the gust diameter is reduced to $30 \mathrm{~m}$ and the spacing to $100 \mathrm{~m}$.

Bethwaite also states that gusts have been measured at $40 \%$ higher wind speed than the average. This was then linearly tapered from $0 \%$ at the edge to $40 \% 7.5 \mathrm{~m}$ from the edge.

\section{RESULTANT VELOCITY POLARS}

Using the model described above the performance of the simulator has been estimated by simply trying it out. That is, an optimum sailing condition was obtained by testing different mainsheet angles and different heel angles. The performance was measured assuming that a $75 \mathrm{~kg}$ sailor was using the simulator and was able to obtain the optimum heel angle. The results are shown in Figure 13.

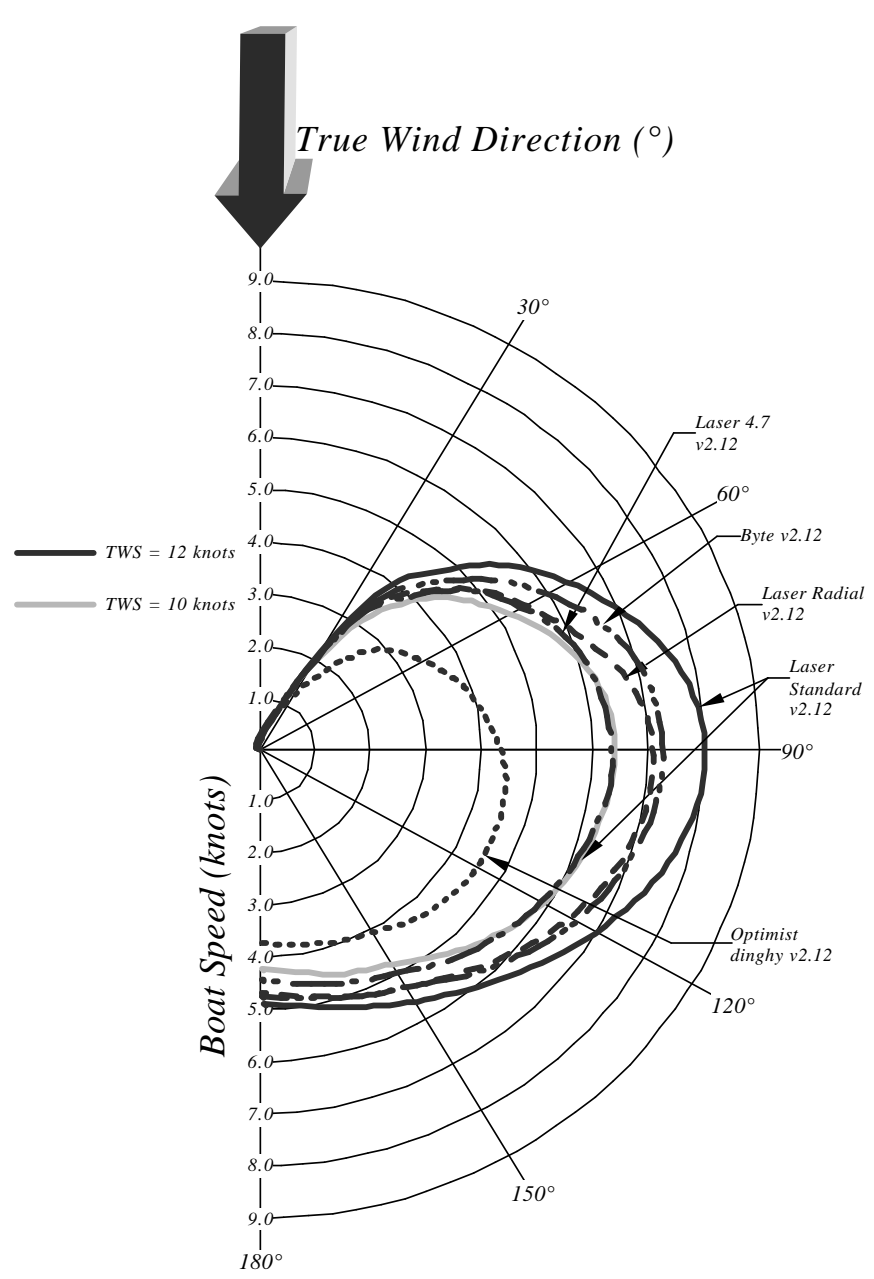

Figure 13: Polar plots for the current simulator performance

Figure 13 shows the estimated velocities for each simulation model. This is a polar plot whereby the angle away from the vertical, in a clockwise direction, shows the angle of the boat relative to the wind. The distance away from the origin shows the boat velocity. In Figure 13 two true wind speeds are shown for the standard Laser, all other boats have been estimated for a true wind speed of 12 knots only.

A comparison was made between the simulator predicted sailing performance using the original and new programs. Results are presented in Figure 14. 


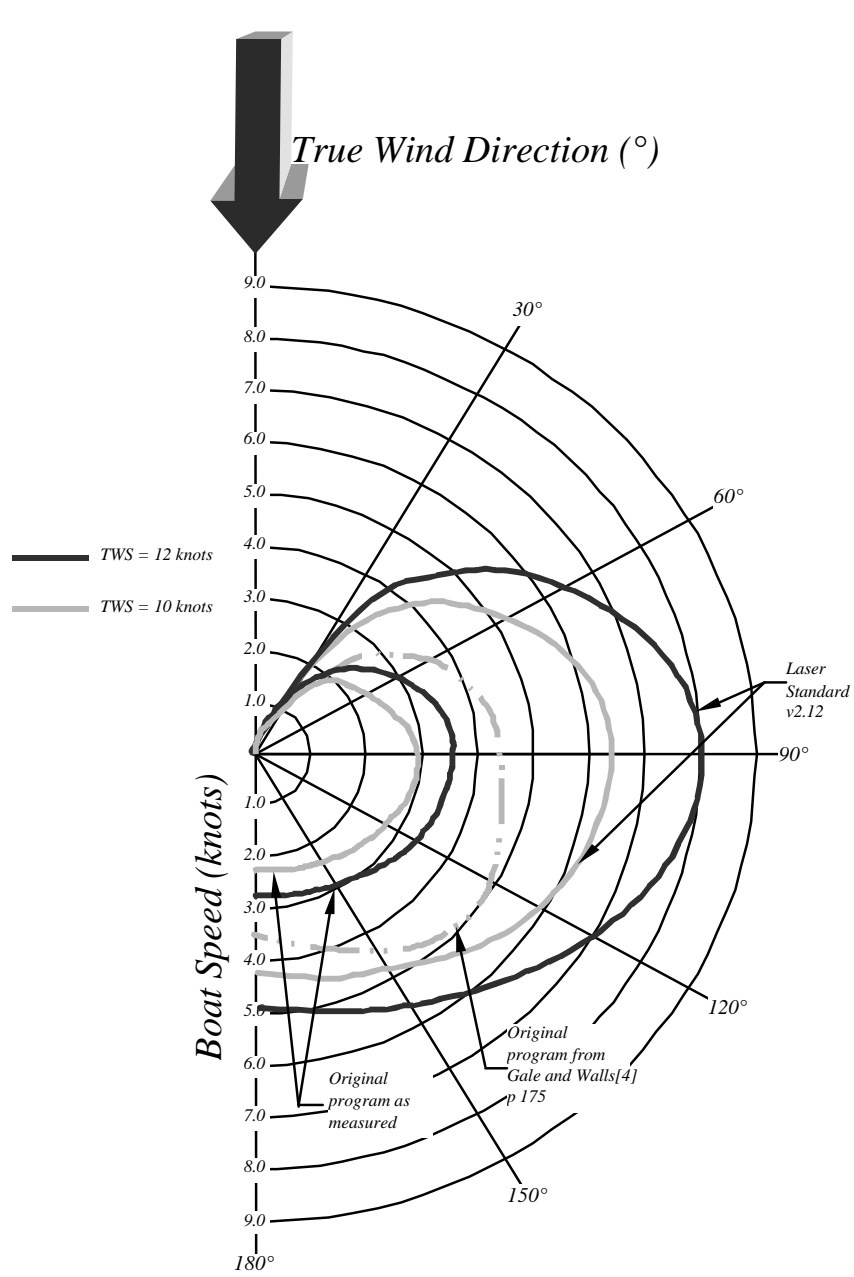

Figure 14: Comparison of original simulator to current simulator performance

There are also presented in Figure 14 some results obtained from Gale and Walls [4] which is a report on the original simulator as developed at the University of Tasmania.

A comparison was also made with some on water measurements made by Frank Bethwaite. This comparison is shown in Figure 15.

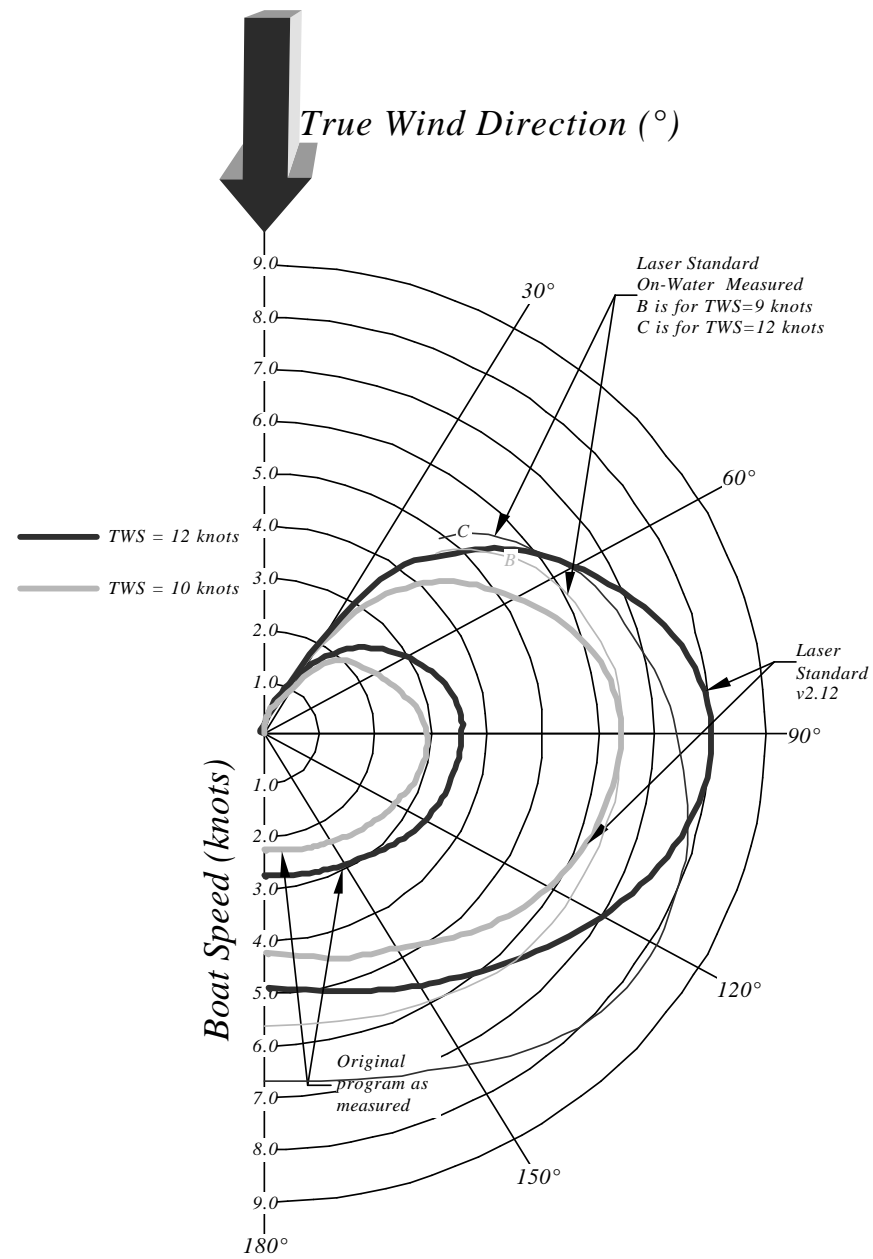

Figure 15: Comparison to on-water measured data

\subsection{Discussion of polar plots}

Taking Figure 15 first, the new simulator clearly has considerably improved performance when compared with the original code. The simulator and actual polars at different wind velocities show good correspondence at all wind angles except downwind, where the simulator polars are slower than the on-water measurements. However this difference between predicted and on-water measurement at a true wind angle of $180^{\circ}$ may be explained by the fact that the on-water measurements were made under surfing conditions.

Assuming the Laser polar is acceptable then Figure 13 can be revisited. It can be seen from this figure that the three boats relate to each other quite well. The Byte is seen to have a slightly better performance than the Laser Radial. This difference becomes slightly larger as the wind strength is increased because the Byte has slightly lower upright drag at higher boat speeds.

Finally Figure 14 can be considered. As mentioned above the new program predicts far greater boat speeds than the original. This is primarily because of the much lower upright drags being used (which is considered 
more realistic). Also the drag values for the lifting surfaces have been reduced significantly (also considered more realistic). Different polar curve shapes are evident in the new program primarily because the new program has an explicit sway force balance. The appropriateness of these higher velocities is supported by the on water measurements.

Also, Figure 14 includes a curve taken from Gale and Walls p 175 [4]. This should have matched the curve of "Original program as measured". Some plausible reasons that it doesn't, being only conjecture, are listed below:

- Gale and Walls used a numerical optimisation procedure to determine sailing performance. Perhaps this has resulted in different sailing conditions, which optimised the physical model being used. The use of an optimisation routine is preferable from a repeatability point of view, however, it has not been used here because of the rudimentary nature of the physical model.

- it is stated in Gale and Walls (pp 173-175) [4] that surf-riding effects have been taken into consideration, this was not implemented in the original program used for comparison with the new program.

Differences between the performance prediction of the two programs are to be expected since the assumptions used for the models were different in important respects, as outlined above. What is more puzzling is that although there was a good fit between the simulator performance and on water data in Gale and Walls, the series of on water data in the paper are most likely too slow for a Laser (Gale and Walls p 175, Figure 9) [4]).

\section{DYNAMIC RESPONSE OF THE NEW PROGRAM}

At this stage the only judgement of the dynamic response of the new program has been by individuals on a subjective basis. However, the individuals have a wealth of experience both with actual and virtual sailing. Reports so far have indicated that the enhancements made to the physical model described in this paper have led to an improved dynamic response of the simulator. Perhaps the best indications of this are seen at very high speeds and very low speeds. For these conditions it was reported by one of the most experienced software testers that the new program "feels" more realistic.

\section{CONCLUSIONS}

The changes to the physical model of the simulator have improved the realism. This improvement can be seen by the polar plots of performance shown in this paper along with the comments made regarding the dynamic response.

The fact that the physical modelling developments have been accompanied by a complete modularisation of the code has meant that future modifications can be performed much more efficiently. For example it is now possible to code an entirely new boat (as long as it fits into the physical model presented here) in three days of coding.

Further development of the simulator is now much more straightforward. Numerous new options are currently being investigated, perhaps the most exciting will be one which will allow two simulators to race against each other. This would allow existing simulators to be incorporated into the same race.

\section{Acknowledgements}

The project so far has been running on a commercial basis, being funded by Virtual Sailing. Jonathan Binns was responsible for programming, assisted and advised by Peter Niekamp (AMC). Norman Saunders contributed ideas for program development and in program evaluation. Frank Bethwaite provided on water data and discussion of the physical model. A simulator was provided by Virtual Sailing Pty Ltd.

\section{References}

1. Bertram, V., Practical Ship Hydrodynamics, 2000, 1st Ed., Butterworth-Heinemann, Oxford, UK, $\mathrm{x}+270$ pages

2. Bethwaite, F., High Performance Sailing, 1993, 1st Ed., International Marine, Camden, USA, 414 pages

3. Binns, J.R., Hull-Appendage Interaction of a Heeled and Yawed Vessel, 1996, Curtin University of Technology, Perth, Australia, xiv +189 pages

4. Gale, T.J. and Walls, J.T., "Development of a Sailing Dinghy Simulator", Simulation, 2000, 74:3, Simulation Councils, Inc., USA, 167-179

5. Gerritsma, J., Keuning, J.A. and Versluis, A., "Sailing Yacht Performance in Calm Water and in Waves", The 11th Chesapeake Sailing Yacht Symposium, 1993, SNAME, Maryland, USA, pp 233-245

6. Lewis, E.V. Ed., Principles of Naval Architecture, Vol III Motions in Waves and Controllability, 1989, 3rd Ed., SNAME, Jersey City, USA, vi + 429 pages

7. Marchaj, C.A., Aero-Hydrodynamics of Sailing, 1988, 2nd Ed., Adlard Coles Nautical, London, UK, xv +743 pages

8. Rae, W.H. and Pope, A., Low-Speed Wind Tunnel Testing, 1984, 2nd Ed., John Wiley \& Sons, New York, USA, xiii + 534 pages

9. Renilson, M. and Manwarring, T., "An Investigation into Roll/Yaw Coupling and its Effect on Vessel Motions in Following and Quartering Seas", The 7th International Conference on Stability of Ships and Ocean Vehicles, 2000, Vol. A, AMECRC, Launceston, Australia, pp 452459

10. Renilson, M.R. and Tuite, A.J., "Broaching Simulation of Small Vessels in Severe Following Seas", AMECRC Report C 95/4, 1995, AMECRC, Launceston, Australia

11. Saunders, H., Hydrodynamics in Ship Design, 1957, Vol. 2, SNAME, New York, USA, xxiii + 980 pages

12. Teeters, J.R., "Refinements in the Techniques of Tank Testing Sailing Yachts and the Processing of Test Data", The 11th Chesapeake Sailing Yacht Symposium, 1993, , SNAME, Annapolis, USA, pp 13 - 33

13. Walls, J.T., Gale, T., Saunders, N.R. and Bertrand, L., "Assessment of upwind dinghy sailing performance using a virtual reality dinghy sailing simulator", The Australian Journal of Science and Medicine in Sport, 1998, Vol. 1, pp 40-52 